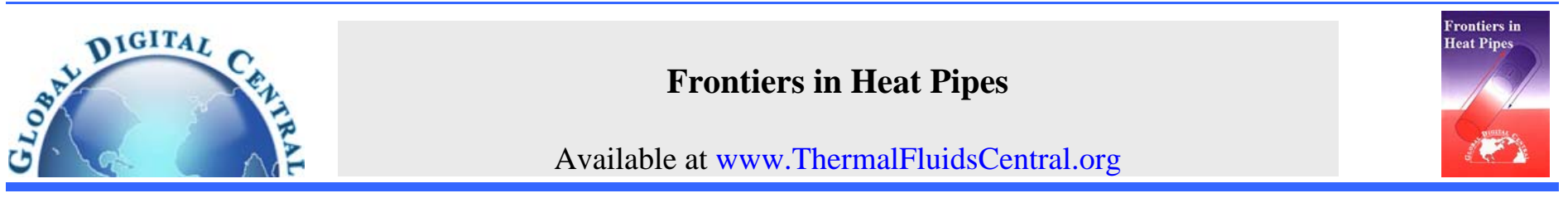

\title{
SIMPLE EVALUATION METHOD FOR EFFECTIVE THERMAL CONDUCTIVITY OF A WICK STRUCTURE FILLED WITH A WORKING FLUID
}

\author{
Yasushi Koito*, Ryo Yamaguchi, Toshio Tomimura \\ Faculty of Advanced Science and Technology, Kumamoto University, Kumamoto-shi, Kumamoto, 860-8555, Japan
}

\begin{abstract}
Effective thermal conductivity of a heat pipe wick structure filled with a working fluid is measured. A simple evaluation method using a heating and a cooling rod is described and the value of effective thermal conductivity is obtained by comparing the numerical results with the experimental ones. A frame and sheets are employed to prevent the leak of the working fluid from the wick structure. Three type of sintered copper powders and water are used as the wick structure and the working fluid, respectively. The measurement results are shown and then compared with the previous empirical equations.
\end{abstract}

Keywords: Thermal design, Heat pipe modeling, Sintered powder.

\section{INTRODUCTION}

In mathematical modeling of a capillary-wick heat pipe, the effective thermal conductivity is often used to calculate the heat transfer through a wick structure (Faghri, 1995; Vadakkan et al., 2004; Koito et al., 2006). Actually, a heat pipe wick is complicated in structure and filled with a working fluid. The use of effective thermal conductivity simplifies the heat transfer calculation of a wick structure filled with a working fluid because they are modeled as a homogeneous medium. The calculation using the effective thermal conductivity is a practical way. However, since the effective thermal conductivity of a wick structure strongly affects the temperature distribution of a heat pipe, its evaluation is important in conducting the thermal design of a heat pipe.

The thermal conductivity of a solid plate, on the other hand, can be measured simply by creating a steady-state one-dimensional temperature distribution in a sample (Tomimura et al., 2011). In this measurement method, a sample is sandwiched between a heating and a cooling rod, and a steady-state one-dimensional temperature distribution of a sample is measured. From the temperature distribution, the value of thermal conductivity of a sample is obtained by using Fourier's law. This measurement method was also applied for the study to evaluate the heat transfer between solid surfaces in contact (Koito et al., 2014).

The present study extends the above-mentioned simple measurement method to evaluate the effective thermal conductivity of a heat pipe wick structure filled with a working fluid. Similar measurement methods were already employed by Xin et al. (2010) and Florez et al. (2013); however, the present study uses a frame and sheets to prevent the leak of a working fluid from a wick structure. Three types of sintered copper powders and water are used as a wick structure and a working fluid, respectively. Experiments and numerical analyses are conducted to determine the value of effective thermal conductivity. Furthermore, because many empirical equations were already proposed to predict the effective thermal conductivity of a wick structure, the comparison is also made between the present measurement results and the previous empirical equations. The previous empirical equations were reviewed by Atabaki and Baliga (2007) in detail.

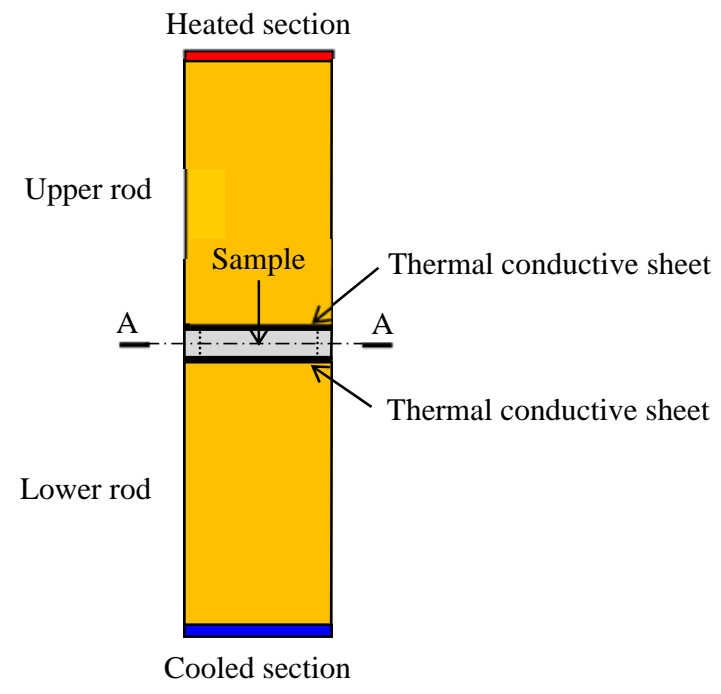

Cross section A - A

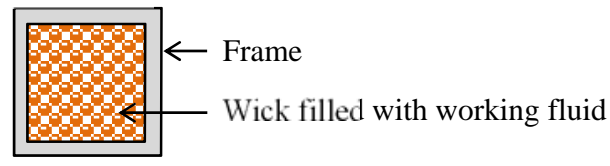

Fig. 1 Physical model

\footnotetext{
* Corresponding author. Email: koito@gpo.kumamoto-u.ac.jp
} 
[6]

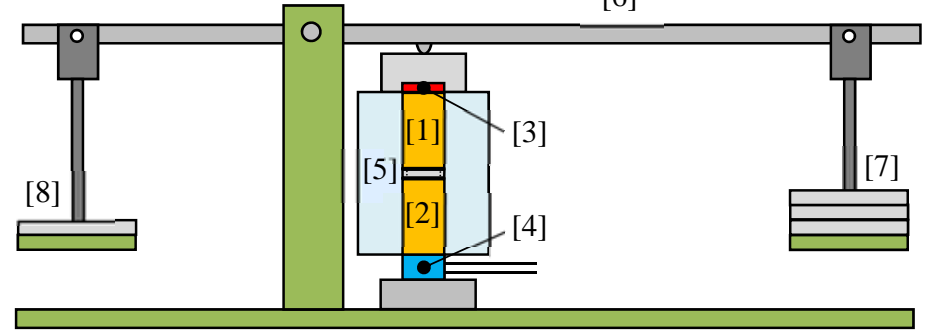

[1] Upper rod

[2] Lower rod

[3] Ceramic heater

[4] Cooling device

[5] Thermal insulator

[6] Lever

[7] Weight

[8] Balance weight

Fig. 2 Schematic diagram of measuring apparatus

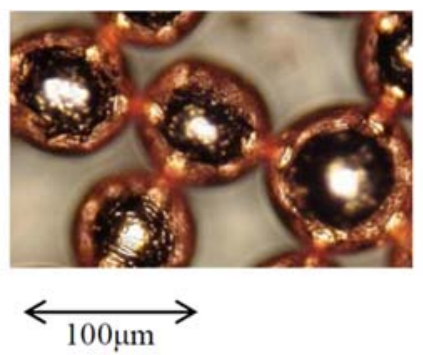

(a) Type 1-a

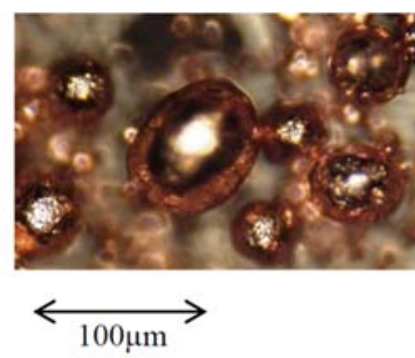

(b) Type 1-b

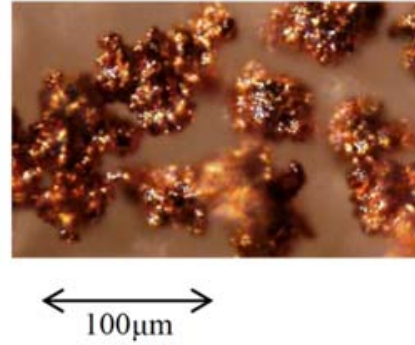

(c) Type 2

Fig. 3 Photograph of copper powders before sintering

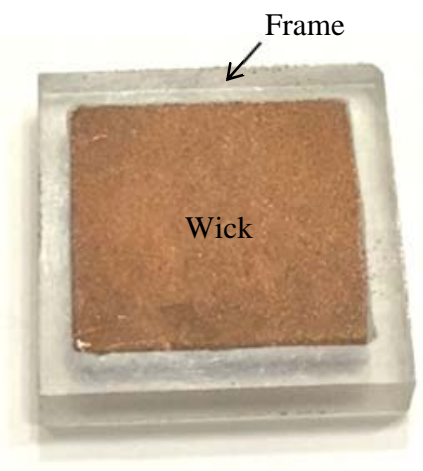

Fig. 4 Photograph of sintered copper powders (Type 1-a)

\section{PRINCIPLE OF MEASUREMENT}

Figure 1 shows a physical model to evaluate the effective thermal conductivity. A sample of a wick structure filled with a working fluid is shown at the center of the model. A plastic frame and two thermal conductive sheets are used to prevent the leak of a working fluid from a wick structure. Then, they are sandwiched between two rods (an upper and a lower rod). The top surface of the upper rod is heated at a heat flux of $q_{h}$, while the bottom surface of the lower rod is kept at a lower temperature of $T_{c}$. The outer surface of the model is adiabatic except for the top surface of the upper rod and the bottom surface of the lower rod.

A steady-state temperature distribution of the model is obtained by conducting the numerical analysis. It is noted that because the effective thermal conductivity of a wick structure filled with a working fluid, $\lambda_{\text {eff, }}$ is not equal to the thermal conductivity of the plastic frame, $\lambda f$, threedimensional constriction and spreading heat flow would be found inside the model. Therefore, the three-dimensional analysis is required and the temperature distribution inside the model is obtained by solving the governing equation of

$$
\nabla \cdot \lambda_{i} \nabla T=0 \quad\left(\lambda_{i}=\lambda_{\text {eff }}, \lambda_{r}, \lambda_{s}, \lambda_{f}\right)
$$

with the boundary conditions:

$$
\begin{array}{ll}
-\lambda_{r}(\partial T / \partial n)=q_{h} & \text { for heated section } \\
T=T_{c} & \text { for cooled section } \\
\partial T / \partial n=0 & \text { for adiabatic section }
\end{array}
$$

where $\lambda_{r}$ and $\lambda_{s}$ are the thermal conductivities of the rod and the thermal conductive sheet, respectively, and $n$ is the coordinate normal to the boundary surface.

When $\lambda_{r}, \lambda_{s}, \lambda_{f}$ are given, the steady-state temperature distribution of the model is obtained numerically with $\lambda_{\text {eff }}$ as a parameter. Therefore, by conducting the experiment to measure the steady-state temperature distribution of the model and then comparing the numerical results with the experimental ones, the value of $\lambda_{\text {eff }}$ is determined.

\section{EXPERIMENTAL METHOD}

Figure 2 shows an experimental apparatus. The apparatus is essentially the same as described in the previous papers (Tomimura et al., 2011; Koito et al., 2014). Brass rods having the size of $25 \mathrm{~mm}$ (depth) $\times 25$ $\mathrm{mm}$ (width) $\times 45 \mathrm{~mm}$ (height) were used as the upper and the lower rod. A ceramic heater was employed to heat the top surface of the upper rod, while the bottom surface of the lower rod was water-cooled by using a small heat exchanger. The dimension of the heater was $25 \mathrm{~mm}$ (depth) $\times 25 \mathrm{~mm}$ (width) $\times 1 \mathrm{~mm}$ (thickness) and the cooling water was supplied from a thermostatic bath. The material of a frame was polycarbonate. The dimension of the inner space of the frame was $19 \mathrm{~mm}$ (depth) $\times 19$ $\mathrm{mm}$ (width) and its height was equal to the thickness of a wick structure. 


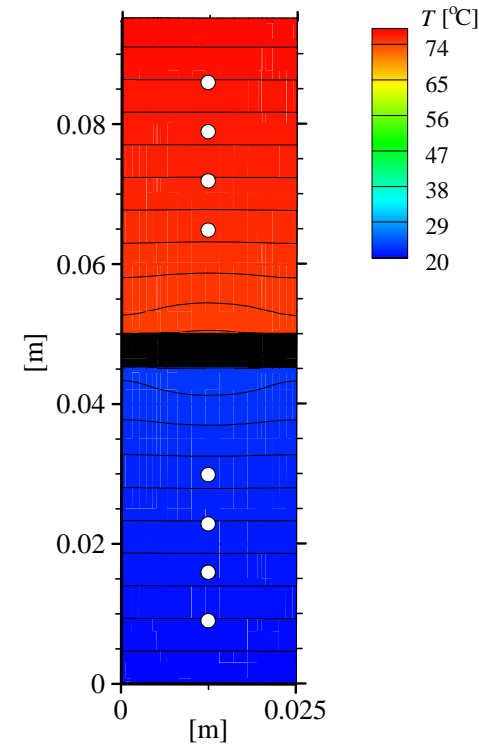

(a) $\lambda_{\text {eff }}=1.0 \mathrm{~W} /(\mathrm{m} \cdot \mathrm{K})$

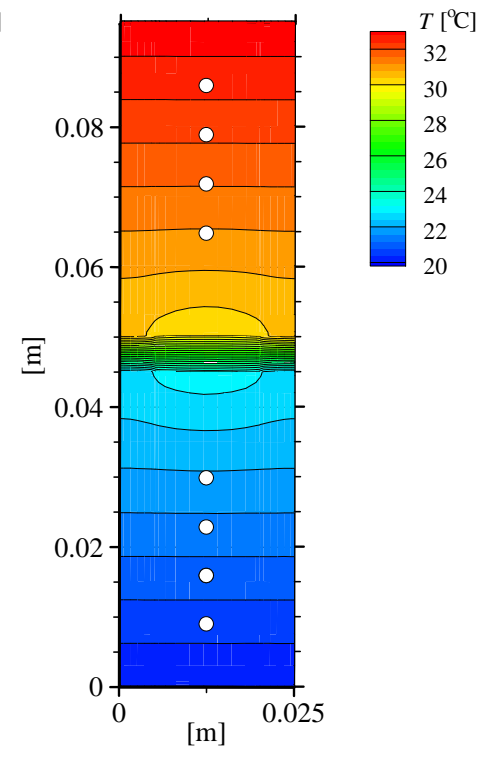

(b) $\lambda_{\text {eff }}=10 \mathrm{~W} /(\mathrm{m} \cdot \mathrm{K})$

Fig. 5 Numerical results of temperature distribution inside rods

A wick structure and a working fluid were filled in the frame, and then they were sandwiched between two thermal conductive sheets to prevent the leak of a working fluid. A lever and weights were used to load a constant pressure on the upper and the lower rod. Moreover, the two rods and the frame were covered with a thermal insulator.

In experiment, the heat flux at the top surface of the upper rod, $q_{h}$, and the temperature at the bottom surface of the lower rod, $T_{c}$, were kept at $q_{h}=8000 \mathrm{~W} / \mathrm{m}^{2}$ and $T_{c}=20^{\circ} \mathrm{C}$, respectively. The temperature distributions inside the upper and the lower rod were measured by thermocouples. The measuring points of temperatures were $9 \mathrm{~mm}, 16$ $\mathrm{mm}, 23 \mathrm{~mm}$ and $30 \mathrm{~mm}$; they were positioned from the top of the upper rod and also from the bottom of the lower rod. Small holes were drilled on each rod and T-type thermocouples of $0.5 \mathrm{~mm}$ in diameter were inserted by applying a thermally conductive silver paste. Uncertainly in measuring the temperature was $0.1{ }^{\circ} \mathrm{C}$. Before conducting the experiment, the thermal conductivities of the rod, $\lambda_{r}$, the thermal conductive sheet, $\lambda_{s}$, and the frame, $\lambda_{f}$, were measured, and $\lambda_{r}=124$ $\mathrm{W} /(\mathrm{m} \cdot \mathrm{K}), \lambda_{s}=2.99 \mathrm{~W} /(\mathrm{m} \cdot \mathrm{K})$ and $\lambda_{f}=0.45 \mathrm{~W} /(\mathrm{m} \cdot \mathrm{K})$ were obtained.

As shown in Fig. 3, three types (Type 1-a, Type 1-b, Type 2) of copper powders were used in the present study. Type 1-a and Type 1b were almost sphere in shape, but smaller copper powders were also included in Type 1-b. The powder size distribution of Type 1-b was wider than that of Type 1-a. The shape of Type 2, on the other hand, was irregular. The copper powders were sintered by using a furnace, and then set inside the frame as shown in Fig. 4. The thicknesses of the sintered copper powders of Type 1-a and Type 1-b were $5.0 \mathrm{~mm}$, and that of Type 2 was $4.5 \mathrm{~mm}$. Moreover, the porosities, $\varepsilon$, of Type 1-a, Type 1-b and Type 2 were $38 \%, 36 \%$ and $52 \%$, respectively. Water was used as the working fluid.

\section{RESULTS AND DISCUSSION}

Figure 5 shows the numerical results of the steady-state temperature distribution inside the model. The numerical results for $\lambda_{\text {eff }}=1.0$ $\mathrm{W} /(\mathrm{m} \cdot \mathrm{K})$ and $\lambda_{\text {eff }}=10 \mathrm{~W} /(\mathrm{m} \cdot \mathrm{K})$ are shown in this figure. The numerical calculation was performed under the same heating/cooling conditions as prescribed in the experiment, namely $q_{h}=8000 \mathrm{~W} / \mathrm{m}^{2}$ and $T_{c}=20^{\circ} \mathrm{C}$. The governing equation was discretized by using a control volume

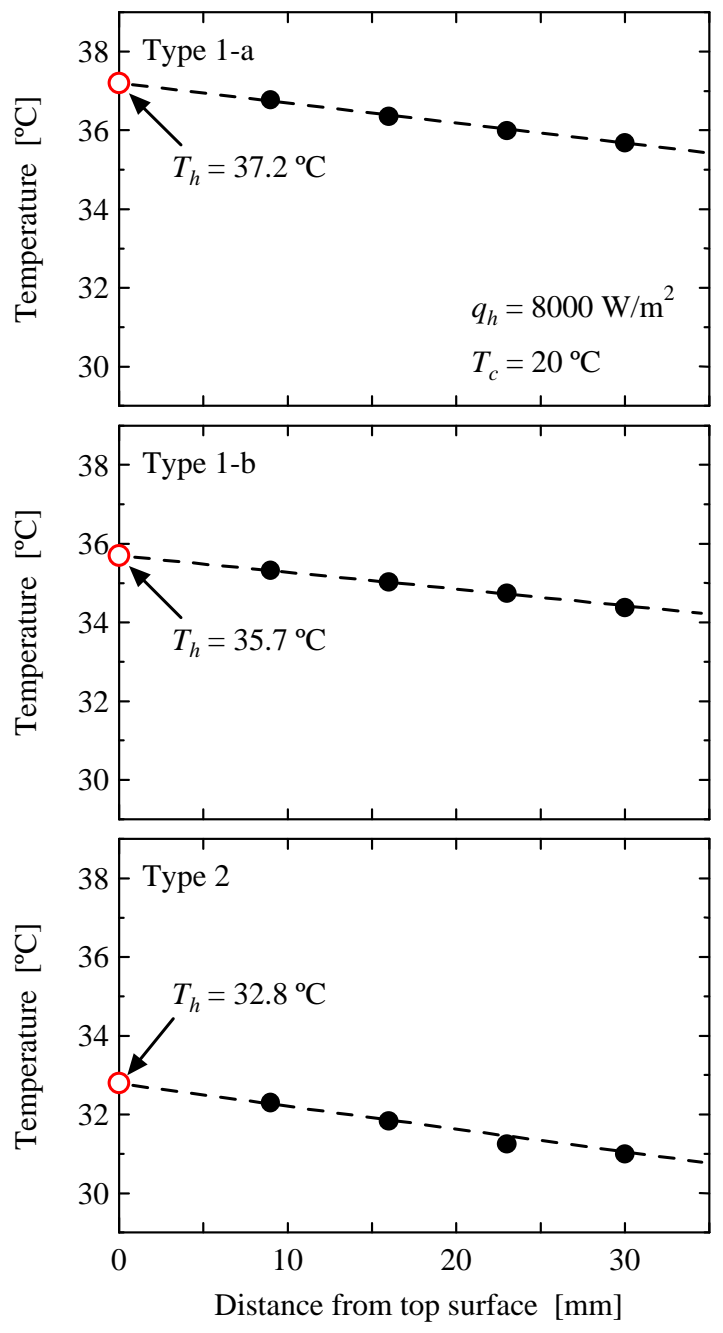

Fig. 6 Experimental results of temperature distribution inside upper rod

method (Patankar, 1980). The computational program to solve the discretized equations with the boundary conditions was made by the authors. The mesh size was set sufficiently small to meet the energy balance of the computational domain, that is, the heat output from the cooled section agreed with the heat input to the heated section within $0.1 \%$. The measuring points of temperatures in the experiment are also indicated by white circles in this figure. As expected, the temperature difference inside the model for $\lambda_{\text {eff }}=10 \mathrm{~W} /(\mathrm{m} \cdot \mathrm{K})$ was smaller than that for $\lambda_{\text {eff }}=1.0 \mathrm{~W} /(\mathrm{m} \cdot \mathrm{K})$. In both cases, the temperature gradients inside the frame and the wick structure filled with the working fluid were much larger than those inside the rods. Moreover, due to the difference between $\lambda_{\text {eff }}$ and $\lambda_{\text {f }}$, the constriction and the spreading of heat flow were found inside the upper and the lower rod respectively near the wick structure. In the experiment, to prevent the effect of the constriction and the spreading of heat flow, the thermocouples were set in the rods apart from these heat-flow regions.

Figure 6 shows the experimental results of the steady-state temperature distribution inside the upper rod. The experimental results for Type 1-a, Type 1-b, Type 2 are shown in this figure. As expected, when the heating was started, the temperatures inside the rods increased rapidly and then the temperature changes became smaller with time. In the cases of Type 1-a and Type 1-b, the temperature changes became less than $0.1^{\circ} \mathrm{C}$ in $60 \mathrm{~min}$, while it took $50 \mathrm{~min}$ for Type 2 . It was confirmed that linear temperature distributions were obtained in all cases. Therefore, as shown in each figure by a dashed line, the temperature at 

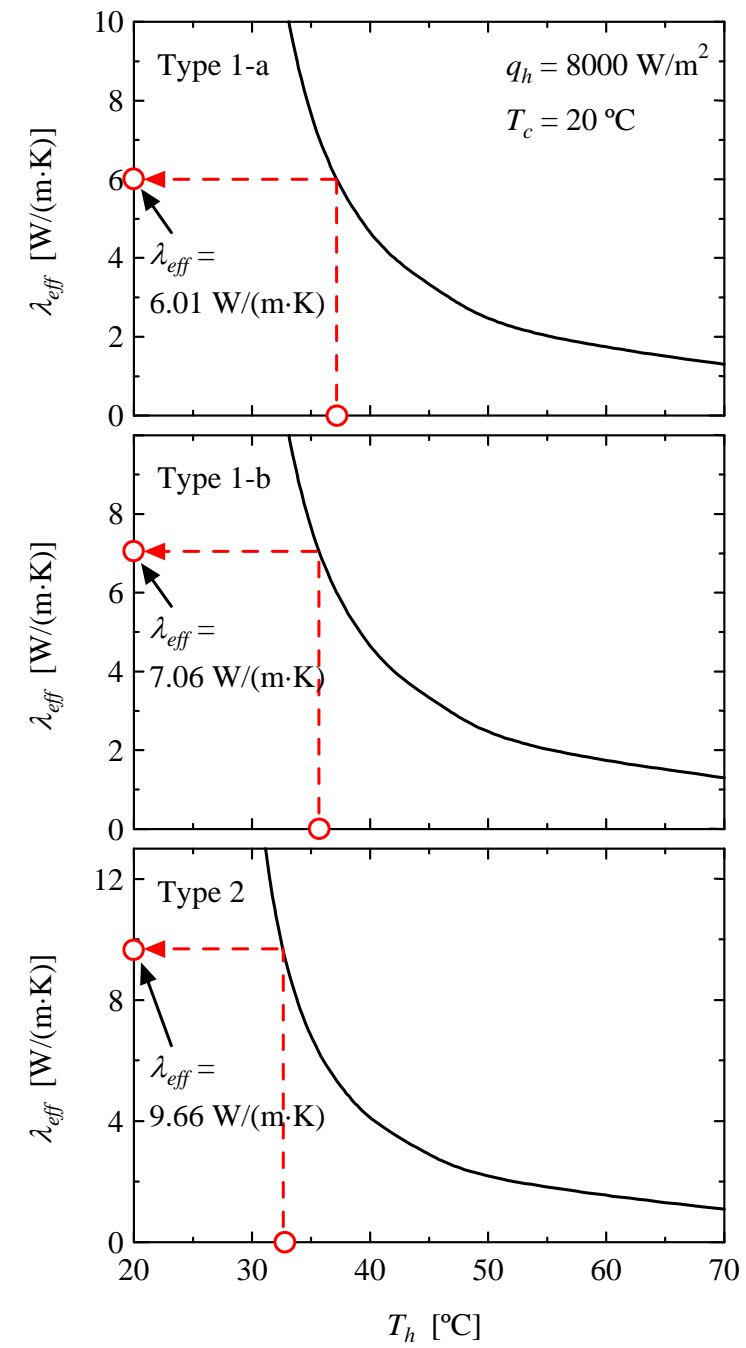

Fig. 7 Numerical results of relation between temperature at heated section and effective thermal conductivity

the heated section, $T h$, was evaluated by extrapolating the temperatures measured at the positions of $9 \mathrm{~mm}, 16 \mathrm{~mm}, 23 \mathrm{~mm}$ and $30 \mathrm{~mm}$ from the top of the upper rod. The value of $T_{h}$ is also indicated in this figure.

Figure 7 shows the numerical results of the relation between the temperature $T_{h}$ and the effective thermal conductivity $\lambda_{\text {eff }}$. This relation was obtained by conducting the numerical calculation with $\lambda_{\text {eff }}$ as a parameter. It is noted that the numerical result for Type 1-a was equal to that for Type 1-b, but they were slightly higher than that for Type 2. This is due to the difference in the thickness of wick structure, namely $5.0 \mathrm{~mm}$ for Type 1-a and Type 1-b while $4.5 \mathrm{~mm}$ for Type 2 as mentioned above. The numerical results show that $\lambda_{\text {eff }}$ strongly depends on $T_{h}$. By using this relation, the value of $\lambda_{\text {eff }}$ was evaluated from the experimental result of $T_{h}$. As indicated in each figure by a dashed line, the values of $\lambda_{\text {eff }}$ were obtained as $\lambda_{\text {eff }}=6.01 \mathrm{~W} /(\mathrm{m} \cdot \mathrm{K}), \lambda_{\text {eff }}=7.06$ $\mathrm{W} /(\mathrm{m} \cdot \mathrm{K}), \lambda_{\text {eff }}=9.66 \mathrm{~W} /(\mathrm{m} \cdot \mathrm{K})$ for Type $1-\mathrm{a}$, Type $1-\mathrm{b}$, Type 2, respectively.

Figure 8 shows the result of effective thermal conductivity measurement for Type 1-a, Type 1-b, Type 2. Each measurement was repeated three times, and all the results are shown in this figure. Moreover, the calculated values of $\lambda_{\text {eff }}$ by the Yagi-Kunii's equation (JAHP, 2001) and the Alexander's equation (Atabaki and Baliga, 2007) are also shown in the figure. The Yagi-Kunii's equation was given for sintered metal powder wicks and expressed as

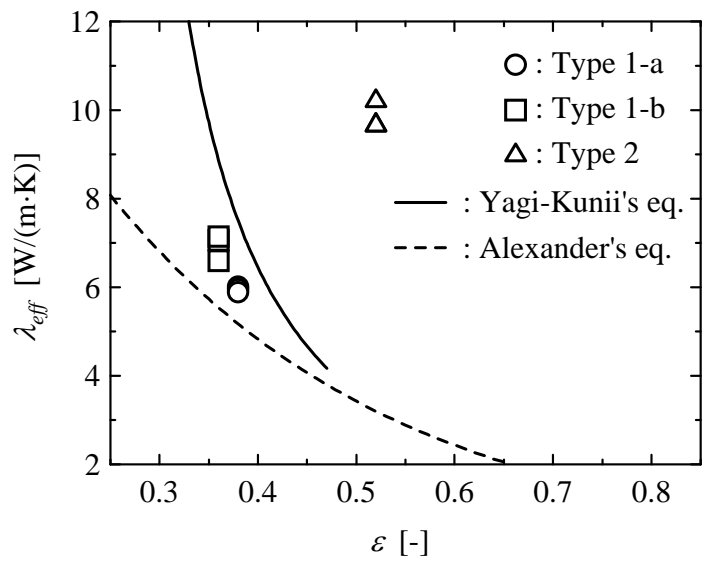

Fig. 8 Effective thermal conductivity of wick structure filled with working fluid

$$
\lambda_{\text {eff }}=\lambda_{l}\left\{\varepsilon+\frac{1-\varepsilon}{\phi+(2 / 3)\left(\lambda_{l} / \lambda_{s}\right)}\right\}
$$

where $\lambda_{l}$ and $\lambda_{s}$ are the thermal conductivities of the liquid and the solid phase, respectively, in the wick structure, $\varepsilon$ is the porosity and $\phi$ the empirical constant accounting for the heat transfer rate between adjacent particles. $\phi$ was expressed as

$$
\begin{aligned}
\phi= & \phi_{2}+\left(\phi_{1}-\phi_{2}\right)(\varepsilon-0.26) / 0.216 \\
\phi_{i}= & \frac{(1 / 2)\{(\kappa-1) / \kappa\}^{2} \sin ^{2} \psi_{i}}{\ln \left\{\kappa-(\kappa-1) \cos \psi_{i}\right\}-\{(\kappa-1) / \kappa\}\left(1-\cos \psi_{i}\right)}-\frac{2}{3 \kappa} \\
& (i=1,2) \\
\psi_{1}= & 54.74^{\circ}, \quad \psi_{2}=22.33^{\circ}, \quad \kappa=\lambda_{s} / \lambda_{l}
\end{aligned}
$$

It is noted that the Yagi-Kunii's equation was given for the range of 0.260 $<\varepsilon<0.476$. The Alexander's equation, on the other hand, was expressed as,

$$
\lambda_{\text {eff }}=\lambda_{l}\left(\lambda_{s} / \lambda_{l}\right)^{(1-\varepsilon)^{\delta}}
$$

where $\delta$ is the parameter and depends on the type of wick structure. For sintered powders,

$$
\delta=0.53
$$

From Fig. 8, it was found that the value of $\lambda_{\text {eff }}$ for Type 1-b was slightly higher than that for Type 1-a. This is because Type 1-b includes smaller copper powders compared to Type 1-a. Small copper powders are positioned between larger copper powders leading to the enhancement of heat transfer between them. The measured values of $\lambda_{\text {eff }}$ for Type 1-a and Type 1-b were located between the calculated values by the YagiKunii's equation and the Alexander's equation. In average, the measured values for Type 1-a and Type 1-b were $20.7 \%$ and $21.9 \%$ smaller respectively than the calculated value by the Yagi-Kunii's equation, while $15.0 \%$ and $25.1 \%$ larger than the Alexander's equation. It was concluded that, in the cases of spherical powders, the fairly good agreement was obtained between the measurement results and the 
previous empirical equations. The value of $\lambda_{\text {eff }}$ for Type 2 , on the other hand, was found to be higher than those for Type 1-a and Type 1-b, implying that contact surface areas and therefore heat transfer rate between powders for Type 2 were larger than those for Type 1-a and Type 1-b. The value of $\lambda_{\text {eff }}$ for Type 2 was also higher than the calculated values by the Yagi-Kunii's equation and the Alexander's equation.

\section{CONCLUSIONS}

The effective thermal conductivity of the heat pipe wick structure filled with the working fluid was measured. The simple physical model was proposed, where the wick structure was filled with the working fluid by using the plastic frame and the two sheets, and they were sandwiched between the upper and the lower rod. The top surface of the upper rod was heated while the bottom surface of the lower rod was cooled, and the temperature distributions were obtained by the experiments and the three-dimensional numerical analyses. The value of the effective thermal conductivity was determined by comparing the numerical results with the experimental ones. Three types (Type 1-a, Type 1-b, Type 2) of sintered copper powders and water were employed as the wick structure and the working fluid, respectively. The powders of Type 1a and Type 1-b were sphere in shape, but Type 1-b also contained smaller powders compared to Type 1-a. The shape of Type 2 was irregular. Small difference was found between the effective thermal conductivities of Type 1-a and Type 1-b. However, it was concluded that, in the cases of spherical powders of Type 1-a and Type 1-b, the fairly good agreement was obtained between the measurement results and the previous empirical Yagi-Kunii's equation and the Alexander's equation. It was also found that the value of $\lambda_{\text {eff }}$ for Type 2 was higher than those for Type 1-a and Type 1-b, implying that contact surface areas and therefore heat transfer rate between powders for Type 2 were larger than those for Type 1-a and Type 1-b.

\section{NOMENCLATURE}

$n \quad$ coordinate normal to boundary surface (m)

$q \quad$ heat flux $\left(\mathrm{W} / \mathrm{m}^{2}\right)$

T temperature $\left({ }^{\circ} \mathrm{C}\right)$

Greek Symbols

$\delta \quad$ parameter

$\varepsilon \quad$ porosity

$\phi \quad$ empirical constant

$\lambda \quad$ thermal conductivity $(\mathrm{W} /(\mathrm{m} \cdot \mathrm{K}))$

$\begin{array}{ll}\text { Subscripts } & \\ c & \text { cooled section } \\ \text { eff } & \text { effective } \\ f & \text { frame }\end{array}$

$\begin{array}{ll}h & \text { heated section } \\ l & \text { liquid phase } \\ r & \text { rod } \\ s & \text { sheet, solid phase }\end{array}$

\section{REFERENCES}

Atabaki, N., and Baliga, B. R., 2007, "Effective Thermal Conductivity of Water-saturated Sintered Powder-metal Plates," Heat and Mass Transfer, 44 (1), 85-99. http://dx.doi.org/10.1007/s00231-007-0229-8

Faghri, A., 1995, Heat Pipe Science and Technology, Taylor \& Francis.

Florez, J. P. M., Mantelli, M. B. H., and Nuernberg, G. G. V., 2013, "Effective Thermal Conductivity of Sintered Porous Media: Model and Experimental Validation," International Journal of Heat and Mass Transfer, 66, 868-878.

http://dx.doi.org/10.1016/j.ijheatmasstransfer.2013.07.088

Japan Association for Heat Pipes (JAHP), 2001, Jitsuyou Heat Pipe, $2^{\text {nd }}$ ed., Nikkan Kogyo Shimbun, Ltd. (in Japanese).

Koito, Y., Imura, H., Mochizuki, M., Saito, Y., and Torii, S., 2006, "Numerical Analysis and Experimental Verification on Thermal Fluid Phenomena in a Vapor Chamber," Applied Thermal Engineering, 26(1415), 1669-1676.

http://dx.doi.org/10.1016/j.applthermaleng.2005.11.012

Koito, Y., Tsurumoto, M., Hatano, F., and Tomimura, T., 2014, "Heat Transfer between Solid Surfaces in Contact Having a Relatively Large Gap (External Thermal Resistance of a Heat Pipe)," Frontiers in Heat Pipes, 5, 013006.

http://dx.doi.org/10.5098/fhp.5.6

Patankar, S. V., 1980, Numerical Heat Transfer and Fluid Flow, Hemisphere Pub. Corp.

Tomimura, T., Shiotsu, Y., Koito, Y., Ishizuka, M., and Hatakeyama, T., 2011, "Evaluation of Effective Thermal Conductivity of Multilayer Printed Circuit Board," AJTEC2011-44232, Proceedings of the ASME/JSME 2011 8th Thermal Engineering Joint Conference, Honolulu, HI.

Vadakkan, U., Garimella, S. V., and Murthy, J. Y., 2004, "Transport in Flat Heat Pipes at High Heat Fluxes from Multiple Discrete Sources," Journal of Heat Transfer, 126(3), 347-354. http://dx.doi.org/10.1115/1.1737773

Xin, G., Cui, K., Zou, Y., and Cheng, L., 2010, "Reduction of Effective Thermal Conductivity for Sintered LHP Wicks,” International Journal of Heat and Mass Transfer, 53(13-14), 2932-2934. http://dx.doi.org/10.1016/j.ijheatmasstransfer.2010.02.056 